\title{
The medico-legal investigation of sudden, unexpected and/or unexplained infant deaths in South Africa: where are we-and where are we going?
}

\author{
L. du Toit-Prinsloo ${ }^{1}$, J. J. Dempers ${ }^{2}$, S. A. Wadee $^{2}$ and G. Saayman ${ }^{1}$ \\ (1) Department of Forensic Medicine, University of Pretoria, P.O. Box 2034, Pretoria, 0001, South \\ Africa \\ (2) Division of Forensic Medicine and Pathology, National Forensic Pathology Service, University of \\ Stellenbosch, Stellenbosch, South Africa \\ Correspondence author: L. du Toit-Prinsloo \\ Email: lorraine.dutoit@up.ac.za
}

\begin{abstract}
:
Purposes: Sudden Infant Death Syndrome (SIDS) has been reported to be the leading cause of death in infants under one year of age in many countries. Unfortunately, a paucity of published research data exists in South Africa, with regard to the incidence of and investigation into the circumstances surrounding Sudden Unexplained Deaths in Infants (SUDI) and/or SIDS. Currently, even though most academic centers conform to a protocol consistent with internationally accepted standards, there exists no nationally accepted infant death investigation protocol in South Africa. It is the aim of this study to review the current practice of infant death investigation in two representative but geographically and demographically distinct centers.
\end{abstract}

Methods: Retrospective case audit over a five-year period (2000 to 2004) was conducted at two large medico-legal mortuaries in Pretoria (Gauteng) and Tygerberg (Cape Town). Case files on all infants younger than one year of age were reviewed. The outcome measures included number of deaths, demographic details and the nature and final outcome of the post mortem examinations.

Results: A total of 512 cases were identified as possible SIDS cases and of these, 171 was classified as SIDS. The study showed marked inter-case and inter-divisional variation in terms of the investigation of infant deaths at the two institutions.

Conclusions: It is envisaged that this study will focus attention on the current lack of usable data regarding sudden/unexplained/unexpected infant deaths in South Africa, and aid in the formulation and implementation of a practical (yet internationally accountable) infant death investigation protocol, which could facilitate comparisons with other countries and initiate further structured research in this field.

\section{Keywords:}

Sudden infant death syndrome

Sudden unexpected death in infants

Post mortem examination

Cause of death 


\section{Introduction}

The Sudden Infant Death Syndrome (SIDS) has been reported to be the leading cause of death in infants younger than one year of age in most Western countries and in some developing countries [1]. SIDS is most recently defined as "the sudden and unexpected death of an infant under one year of age, with onset of the lethal episode apparently occurring during sleep, that remains unexplained after a thorough investigation including performance of a complete autopsy, and review of the circumstances of death and the clinical history"[2].

Many countries have a national/regional protocol for the investigation of Sudden Unexplained Death in Infants (SUDI) and possible SIDS cases [3]. It is mandatory that a thorough, universally acceptable protocol is necessary in order to examine these infant deaths [2, 3]. These national/regional protocols highlight the importance of all three components necessary for the investigation of sudden, unexpected and/or unexplained infant deaths(possible SIDS cases): review of clinical history; examination of the scene of death; and a thorough post mortem examination augmented by appropriate special investigations [2-5]. The protocols also indicate that resources (for example human and financial) will determine how thoroughly these cases will be investigated. An example of such a protocol is the Dundee Protocol [6]. This protocol consists of investigation of the scene of death, review of clinical history, macroscopic post mortem examination and special investigations, which include cytogenetics, bacteriology, virology, biochemistry, histology, toxicology and radiographs.

The value of death scene investigation is important in determining risk factors for SIDS [7, 8]. Death scene investigation may also elucidate the mechanism of death in some instances, which includes accidental suffocation and wedging [14].

Opinion varies with regards to the value of special investigations. With regards to the value of post mortem bacteriology, some studies have revealed that bacteriology is not very helpful in establishing the cause of death because post mortem bacterial overgrowth only cultures contaminants, especially from specimens obtained from throat swabs [6]. Other publications stress the value of bacteriology and show that even five days' post mortem positive blood cultures reveal the organism that caused death [9]. Screening investigations for virology seem to be more reliable than for bacteriology, since post mortem contamination does not influence the results [10].

Unfortunately, there is a paucity of published research data in South Africa with regard to the incidence of and investigation into SUDI or possible SIDS. Neither does there exist a nationally accepted infant death investigation protocol in South Africa.

This study aims to review the incidence of infant deaths at two large medico-legal mortuaries in South Africa, with regard to the demographical details and the current practice of infant death investigation, as well as the logistical aspects that play a role in the individual divisional policies. The ultimate aim is to introduce an investigative protocol that could be nationally prescribed and implemented, to ensure that more reliable data are generated regarding this enigmatic entity.

\section{Methods}

In South Africa, in instances where an infant had an underlying medical condition/disease and received treatment, the death may be regarded as a natural death in terms of the Birth and Death Registration Act 51 of 1992, and the treating medical personnel may issue a death notification form in order for the death to be registered with the Department of Home Affairs. The body is admitted to a funeral parlor before burial. Other than natural deaths, including sudden/unexplained deaths, are investigated in terms of the Inquests Act 58 of 1959 and these bodies are admitted to a medico-legal mortuary for further investigation. From this it is 
apparent that the admission or not of an infant to a medico-legal facility, is very often dependant on the opinion of the clinician as to whether a disease process had contributed or caused death.

A multicentre audit was done of all case files of deaths of infants younger than one year of age that were admitted to the medico-legal mortuaries of Pretoria and Tygerberg for a fiveyear period from 2000 to 2004. These facilities were selected as they are seen to be representative of large academic facilities, yet are geographically and demographically distinct. At both institutions, the total number of cases (case load) admitted was recorded as well as the total number of infant deaths admitted. The percentage that infants represented in terms of the total case load was determined at each institution.

The history available on all case files upon admission to the mortuaries was reviewed and the infants at the two institutions were divided into three groups: deaths other than natural (unnatural); obvious natural deaths (those with a clear history of a recent illness) and sudden, unexpected and/or unexplained deaths in infants (SUDI, possible SIDS). The available history of the possible SIDS cases usually included an infant with no underlying medical condition and no history of a recent illness who was put down to sleep and who was discovered dead. No specific risk factors were assessed in the study.

On all possible SIDS cases demographic detail as well as the death scene investigation, and the nature and final outcome (cause of death) of the post mortem examination were obtained. The nature of the post mortem examination was divided into macroscopic post mortem examination and additional investigations. The macroscopic post mortem examination was classified into four categories:

- Full macroscopic post mortem examination consisted of evisceration of the body with dissection of all organ blocks;

- Limited post mortem examination consisted of a partial evisceration and dissection (where the process was stopped once an adequate cause of death was ascertained);

- Cases where only an external examination of the body was performed were classified as so called "viewings"; and

- No post mortem examination was done on cases where the history suggested a known underlying disease. In these cases where the death notification form was completed by the treating medical personnel or the pathologist, based on the strength of the clinical history.

The additional investigations included histology, toxicology, bacteriology, virology, biochemistry, genetic studies (to include genetic abnormalities such as SCN5A mutations associated in prolonged QT-syndrome), metabolic studies (to include screening for amongst others medium chain fatty acid disorders) and radiology.

All case files on non-viable products of conception, still births and abandoned babies were excluded from the study.

The number of SUDI or possible SIDS cases and the number of infant deaths concluded as SIDS were determined. The data obtained from the two mortuaries were compared to highlight the differences/similarities in the incidence and depth of investigation of these cases and the final outcome (cause of death).

The data were collected on a data sheet and transferred to a Microsoft Excel spread sheet. The data were transferred to the STATA $10^{\circledR}$ program and analysed in conjunction with a statistician.

Ethics Committee approval was obtained from the Faculty of Health Sciences ethics committees at the Universities of Pretoria and Stellenbosch prior to the commencement of the 
study. Furthermore, consent was obtained from the mortuary managers at the Pretoria and Tygerberg Medico-Legal Mortuaries as well as from the Gauteng Department of Health and the Western Cape Department of Health.

\section{Results}

The total number of infants younger than one year of age admitted to the two medico-legal mortuaries was 813.The total number of cases (case load) admitted to the Pretoria MedicoLegal Mortuary was 12181 with 344 (2.58\%) infants younger than one year of age. At the Tygerberg Medico-Legal Mortuary a slightly higher case load of 13775 cases were admitted over the five year period with 469 (3.40\%) infants younger than one year of age.

The history obtained of the infants admitted to the two institutions was reviewed and SUDI or possible SIDS cases were identified. SUDI (including possible SIDS cases) cases identified were 99 of the 344 cases in Pretoria (28.78\%) and 413 of the 469 cases in Tygerberg (88.06\%), with a combined total of 512 cases. These 512 cases of possible SIDS deaths at the two mortuaries were the population of this study.

The demographical details on the possible SIDS cases were as follows:

Gender:

Pretoria 53 males (53.21\%) and 46 (46.76\%) females.

Tygerberg 214 males (51.94\%), 198 female (48.06\%) and the gender on 1 infant not retrieved.

Age of the infants: (refer to figure 1)

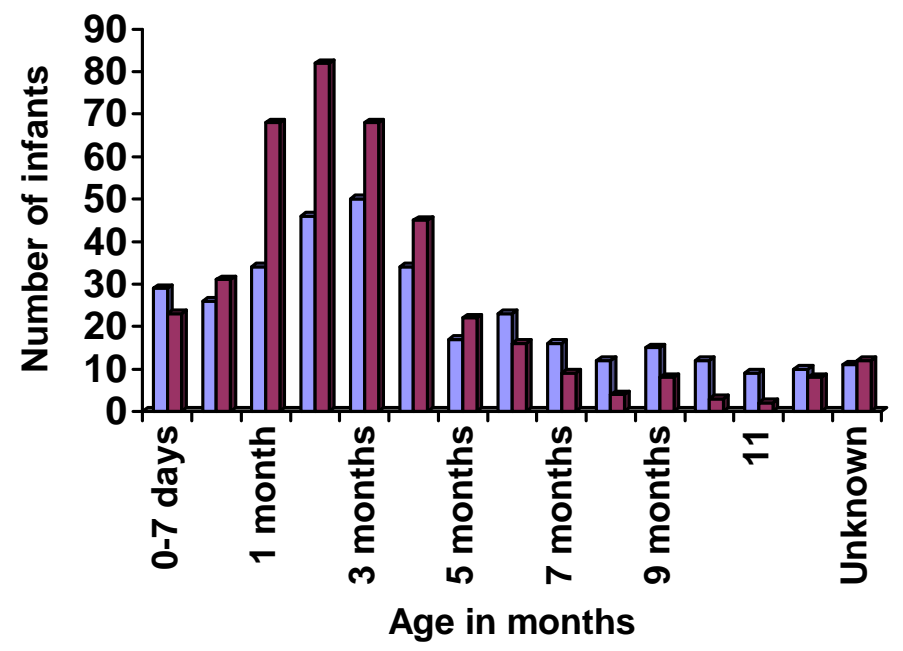

\section{Figure 1: Age of infants}

Racial distribution of the infants: (refer to figure 2) 


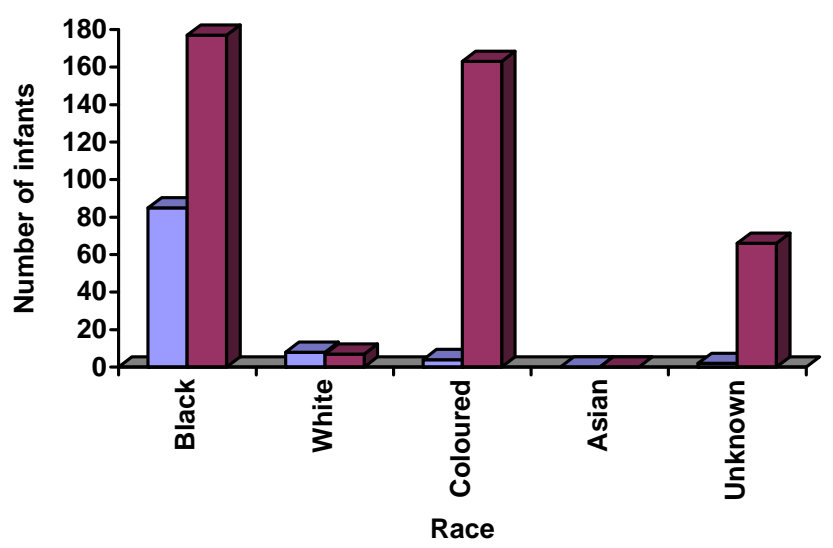

\section{Figure 2: Racial distribution of infants}

Month in which the death occurred: (refer figure 3)

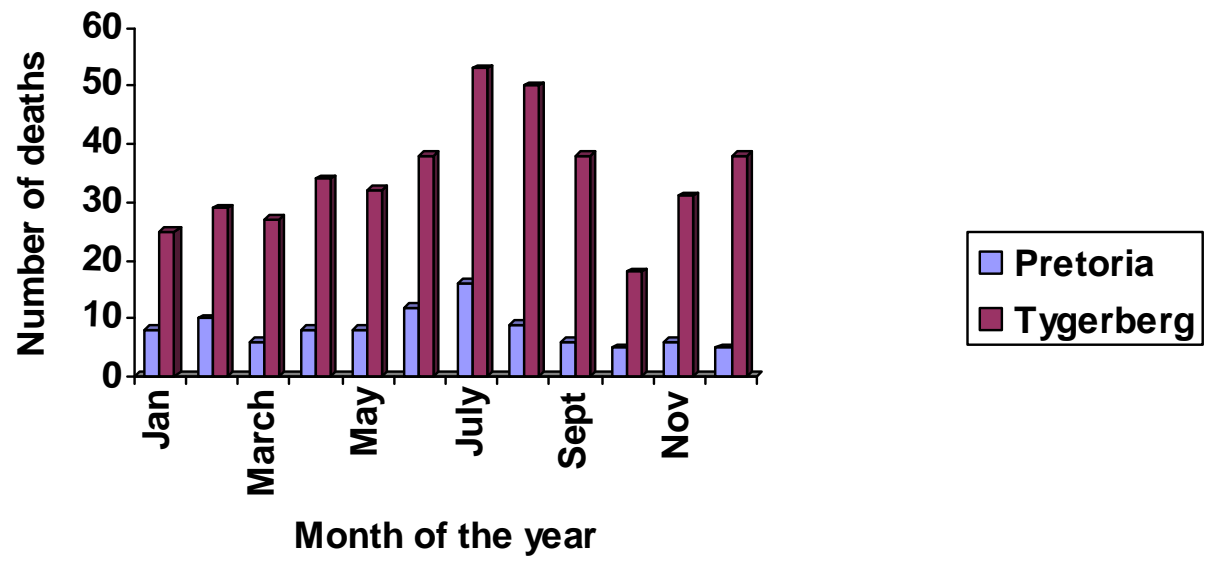

Figure 3: Month in which death occurred

The demographic details showed the following: The peak age at death was between $2-4$ months of age (refer figure 1 above). The racial distribution showed that either more infant deaths occurred amongst the black infants, or more infant deaths are reported / referred to both institutions, and a high number of deaths were present amongst the coloured population in the Tygerberg mortuary. Most deaths occurred in the winter months (June to August) with a second peak in December.

Investigation of death:

Review of the available history upon admission to the respective mortuaries identified/classified 99 infants as possible SIDS cases in Pretoria (with the total number of infants admitted 344) and 413 possible SIDS cases in Tygerberg (with the total number of infants admitted 469). These findings indicated a significantly higher proportion of sudden, unexpected and/or unexplained infant deaths in Tygerberg in comparison to Pretoria. 
Deaths scene investigation was not done on any of the 512 deaths at either the institutions.

The nature of the post mortem examination consisted of the macroscopic post mortem examination (see Table I below) and the additional investigations (see figure 4 below).

Table I: Macroscopic post mortem examination of possible SUDI/SIDS cases

\begin{tabular}{|l|l|l|}
\hline & Pretoria & Tygerberg \\
\hline $\begin{array}{l}\text { Full macroscopic post } \\
\text { mortem examination }\end{array}$ & $60(62.39 \%)$ & $403(97.58 \%)$ \\
\hline $\begin{array}{l}\text { Limited post mortem } \\
\text { examination }\end{array}$ & $19(18.35 \%)$ & $1(0.24 \%)$ \\
\hline Viewing only & $12(11.01 \%)$ & $9(2.18 \%)$ \\
\hline No post mortem examination & $8(8.26 \%)$ & $0(0 \%)$ \\
\hline
\end{tabular}

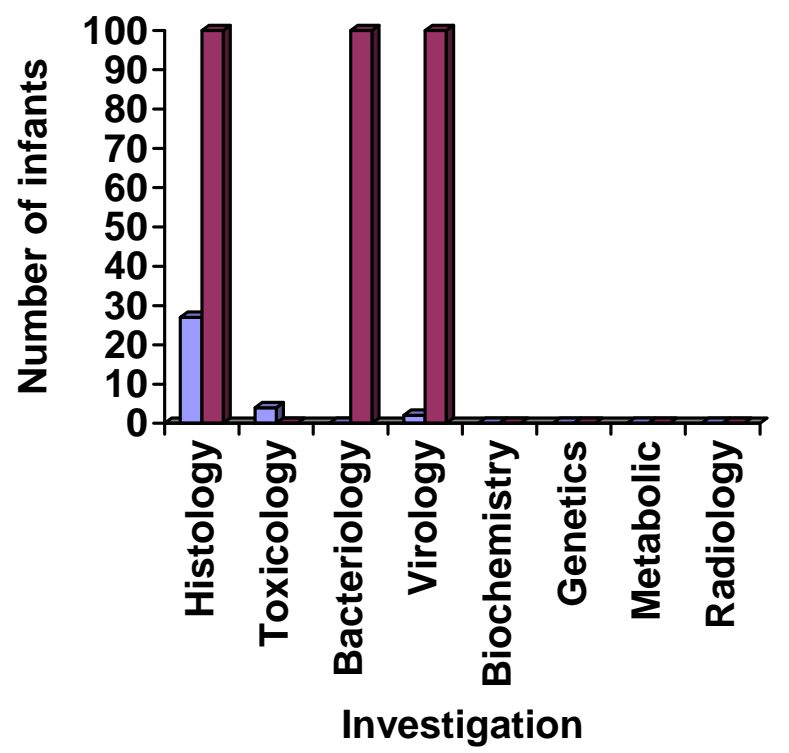

\begin{tabular}{|l|}
\hline Pretoria \\
$\square$ Tygerberg \\
\hline
\end{tabular}

Figure 4: Additional investigations performed

Histological examination of tissue slides were done on more cases in Tygerberg (81.60\%) than Pretoria (29.36\%). Toxicological analysis (which included a general screening for sedatives and analgesics) were done on $6.42 \%$ of the cases admitted to Pretoria, but on none of the cases admitted to Tygerberg. Tygerberg conducted more bacteriology (60.05\%) and virology (59.56\%) screening investigations in comparison to Pretoria (bacteriological examination was not done on any of the cases and virology screening investigation only on $2.75 \%$ of the cases).

Cause of death (in cases presenting as possible SIDS)

Upon completion of the investigations on the possible SIDS cases the final causes of death was concluded to be the following (see table II below) 
Table II: Cause of death

\begin{tabular}{|l|c|c|}
\hline Cause of death & Pretoria & Tygerberg \\
\hline$\underline{\underline{S I D S}}$ & $\underline{10}$ & $\underline{161}$ \\
\hline Unascertained & 12 & 22 \\
\hline Pneumonia & 70 & 129 \\
\hline Other & 7 & 13 \\
\hline Data unavailable (lost) & - & $\mathbf{4 1 3}$ \\
\hline Total & $\mathbf{9 9}$ & \\
\hline
\end{tabular}

SIDS was diagnosed as the cause of death in 10 (9.17\%) of the cases in Pretoria and on 161 $(40.25 \%)$ of the cases in Tygerberg. The most common cause of death was pneumonia (which included bronchopneumonia and interstitial pneumonia) in Pretoria (72.48\%) and SIDS was the leading cause of death in Tygerberg (40.25\%). The deaths indicated as "other" in table II above included mainly infectious diseases such as meningitis and gastroenteritis as well as congenital abnormalities and prematurity associated conditions.

\section{Discussion}

Sudden Infant Death Syndrome (SIDS) is defined as "the sudden and unexpected death of an infant under one year of age, with onset of the lethal episode apparently occurring during sleep, that remains unexplained after a thorough investigation including performance of a complete autopsy, and review of the circumstances of death and the clinical history [2]". It is generally stressed by authors that this is a diagnosis by exclusion which can only be made if all three components (scene investigation, post mortem examination and review of the clinical history) is adhered to in the investigation of possible SIDS cases, although Krous et al did attempt a "layering" of the diagnosis based on the presence or not of scene investigation and special investigation [2]. The value of a thorough investigation protocol in the investigation of sudden, unexpected and/or unexplained infant deaths is important [2-6]. Currently, no specific nationalized protocol exists in South Africa for the investigation of sudden unexplained/unexpected deaths in infants.

This study showed a slightly higher total case load (number of deaths admitted to the respective mortuaries) at the Tygerberg Medico-Legal Mortuary (13 775 compared to 12181 in Pretoria). The total number of infants under 1 year of age was only slightly higher in Tygerberg than in Pretoria (3.40\% of the total case load in Tygerberg and 2.58\% in Pretoria). There were a significantly higher number of possible SIDS cases admitted to the Tygerberg Medico-Legal Mortuary (88.06\%) in comparison to Pretoria (28.78\%).

The demographic details of the infants showed similarities at both institutions. The gender distribution showed a slight male preponderance at both institutions [51.94\% at Tygerberg and 53.21\% at Pretoria $(\mathrm{Pr}=0.814)]$. Peak age at death was consistent with the trend reported in the international literature [13]. Interpretation of the results regarding the racial distribution posed a problematic area. The results gives shows that most deaths were in black infants and in Tygerberg the second highest were in coloured infants. The exact numbers shows a minimal difference between these race groups (black and coloured) in Tygerberg, but the 
normal population of these races differ. Minimal deaths were recorded amongst white infants, and the exact reason could not be ascertained in this study. It should be noted that the racial distribution in the study do not correlate with the racial distribution in the country. This is something that has to be addressed with further research. Most deaths occurred in the winter months (June to August) with a second peak in December month (this does not correlate with international trends and the exact reason could also not be explained in the study).

The pathologist or investigator did not attend any of these deaths scenes at either of the institutions. Research have shown conclusively that death scene investigation may assist in identifying underlying risk factors for SIDS and can also identify cases of wedging or accidental suffocation/strangulation which cannot be proved at post mortem examination [8, 14]. This is important even if the infant was removed from the scene of death and resuscitated or rushed to hospital, as is often the case. In cases where the infant was treated in the emergency room, a doll re-enactment should be done in order to determine the exact position at death and to help identify other possible causes of death.

This study showed similarities as well as variation in the nature of the post mortem examination of SUDI or possible SIDS cases in South Africa, with both inter- and intrainstitutional variation. A full macroscopic post mortem examination was conducted on the majority of cases at both institutions (97.58\% in Tygerberg and 62.39\% in Pretoria). A limited post mortem examination, where the organ dissection was stopped when an adequate macroscopic cause of death was found, was done on more of the cases in Pretoria than Tygerberg (18.35\% in Pretoria and $0.24 \%$ in Tygerberg). Furthermore, a higher number of viewings only were done in Pretoria (11.01\% compared to $2.18 \%$ in Tygerberg). In these cases where viewings were done, additional information in the inquest docket indicated underlying natural disease and South Africa law dictates that a physician may grant a death notification certificate based on good clinical history as well as external examination of the body (according the Birth and Death Registration Act 51 of 1992). At the Pretoria MedicoLegal Mortuary, a number of the cases initially admitted to the mortuary and included in the study population were referred back to the treating physician for completion of the death notification form (8.26\%). The exact reason for this is unclear and it can only be postulated that additional information became available in the inquest docket of the South African Police Service which indicated natural causes, where after the treating physician issued the death notification form. This was not done on any of the 413 infants admitted to the Tygerberg Medico-Legal Mortuary.

Additional investigations included histology, toxicology, bacteriology and virology. No radiological, biochemical, metabolic or genetic testing was performed at either of the institutions. The most probable reason why these tests were not performed is financial (budget) constrains and logistical/resource restraints (samples for genetic testing need to be stored at $-20^{\circ} \mathrm{C}$ and such facilities are not readily available). Histological examination of tissue slides were done on more cases in Tygerberg (81.60\%) than Pretoria (29.36\%). Several publications stress the importance of histological examination in infant deaths [11,12]. Toxicological analyses (which included a general screening for sedatives and analgesics) were done on $6.42 \%$ of the cases admitted to Pretoria, but on none of the cases admitted to Tygerberg. Tygerberg conducted more bacteriology (60.05\%) and virology (59.56\%) screening investigations in comparison to Pretoria (bacteriological examination was not done on any of the cases and virology screening investigation only on $2.75 \%$ of the cases). Some studies strongly suggest that post mortem bacteriology is essential in investigating sudden deaths and states that the death cannot be considered as unexplained if no bacteriology was performed [9].

Upon completion of the investigation the most common cause of death in infants presenting as possible SIDS cases was pneumonia in Pretoria (72.48\%) and consistent with SIDS in 
Tygerberg (40.25\%, 161 infants). SIDS was diagnosed on 10 infants in Pretoria (9.17\%). Pneumonia was the second most common cause of death in the infants in Tygerberg (22.00\%). The term pneumonia refers to both bronchopneumonia and interstitial pneumonia. In many of the cases, the pneumonia/pneumonitis was not classified [15]. Other causes of natural deaths included mostly infectious diseases such as meningitis and gastro-enteritis, but also congenital abnormalities and complications related to prematurity. The congenital abnormalities consisted mostly of congenital cardiac abnormalities with one case of hydrocephalus and one of meningocoele. The complications which were encountered in premature neonates included intra-pulmonary haemorrhages, hyaline membrane disease and necrotizing enterocolitis. With regards to the difference in the number of SIDS cases identified at the two institutions, the possible reasons could be that more SUDI cases are referred to Tygerberg, that pathologists feel more comfortable in making the diagnosis and/or that, Tygerberg has a Mediterranean type climate with cold wet winter months which could have contributed to the added risk of a baby succumbing to an unknown cause and, therefore be classified as SIDS.

The cause of death was signed out as "unascertained" in a number of deaths [34 in total - 12 cases in Pretoria (12.12\%) and 22 cases in Tygerberg (5.33\%)]. This is a term used in cases where the pathologist decides, upon completion of his examination, that the findings of the post mortem examination does not comply with the requirements of an exact diagnoses. The exact reason is unclear and it could only be postulated that the pathologist, being aware of the necessity of additional investigations, did not feel comfortable signing out a case as SIDS if all the special investigations and scene investigation were not done.

In Pretoria, fewer cases were admitted as possible SIDS cases and the reason could lie in the referral system to the mortuary. We postulate that the treating physician, if there is no suspicion of foul play, does not realise SIDS is regarded as an unnatural death, or may want to spare the parents the trauma of having a post mortem examination performed on the baby. Furthermore, the initial review of the admission history to the two mortuaries was done by different observers (pathologists). There could have been a bias in the inclusion in the study with more cases included in Tygerberg and more identified as natural deaths in Pretoria. It should also be noted that Pretoria had a higher number of unnatural deaths $(20.64 \%$ of the cases) with only $11.94 \%$ of the cases admitted to the Tygerberg mortuary being unnatural deaths.

The study showed several limitations. The reason why a number of deaths attributed to "unascertained" remains unclear. Current publications from the South African Department of Statistics indicate the infant mortality rate as 56.2 in 2004 and 46.4 in 2008 [16]. Statistical publications indicate that the total number of infants younger than one year of age that died in 2006 were 47703 with 1292 (2.7\%) of the deaths due to non-natural causes [17]. Currently there are no publications from the Department of Statistics indicating the rate for SIDS. The exact incidence of SIDS therefore, remains unknown. Future research needs to be done, reviewing all infant deaths in South Africa with emphasis on trying to determine the magnitude of the problem of sudden unexpected deaths in infants. This would serve as motivation to introduce an investigative protocol that could be nationally prescribed and implemented, to ensure that these deaths are properly investigated and to generate more reliable data regarding this enigmatic entity.

The recommendations of this study are:

- Further research with emphasis on reviewing all deaths which occurs in children under one year of age (this will include review of all death notification forms at the Department of Home Affairs);

- A campaign to create awareness of the entity amongst parents and to inform them about the investigations which will be required after the loss of an infant; 
- Proper education of health care practitioners (especially general practitioners who work in the emergency departments and paediatricians to refer these cases for medico-legal post mortem examination);

- The design and implementation of a nationalized protocol for the adequate investigation of these cases.

In conclusion, the study demonstrated the demographical profile of infants dying from SIDS to be the same at both institution and in keeping with other international publications [13]. It showed marked variation in the nature of the post mortem examination protocols of SUDI or possible SIDS cases in South Africa, with both inter- and intra-institutional variation. The reasons for the variation are legion, but most certainly need to be addressed in the formulation of a standardised national investigation protocol.

\section{Key points}

1. Sudden Infant Death Syndrome (SIDS) has been reported to be the leading cause of death in infants younger than one year of age in most Western countries and in some developing countries. Currently in South Africa, there are no available statistics on the prevalence of this enigmatic entity.

2. This study shows the demographic profiles of infants dying from SIDS to be in keeping with international publications.

3. The lack of a standardised national investigation protocol for sudden and/or unexpected deaths in infants results in marked variation in the inter- and intrainstitutional investigation of these cases.

4. There seems to be "reluctance" on the part of some of the forensic pathologists in South Africa to certify cases as being consistent with SIDS. In a large proportion of these cases, the cause of death is certified as unascertained.

\section{Acknowledgements}

We would like to thank Prof PJ Becker from the Biostatistics Unit at the MRC in Pretoria for the statistical analysis of the data.

\section{References}

1. Hauck FR, Tanabe KO. International trends in Sudden Infant Death Syndrome: Stabilization of rates requires further action. Pediatrics 2008; 122; 660-666.

2. Krous HF, Beckwith JB, Byard RW, et al. Sudden infant death syndrome and unclassified sudden infant deaths: a definitional and diagnostic approach. Pediatrics 2004; 114; 234-238.

3. Howatson AG. The autopsy for sudden unexpected death in infancy. Current Diagnostic Pathology 2006; 12; 173-183.

4. Krous HF, Byard RW. International standardized autopsy protocol for sudden unexpected infant deaths: appendix I. In: Byard RW, Krous HF, eds. Sudden Infant Death Syndrome: Problems, Progress and Possibilities. London, UK: Arnold; 2001. pp319-333.

5. Corey TS, Hanzlik R, Howard J et al. A functional approach to Sudden Unexplained Infant Deaths. American Journal of Forensic Medicine and Pathology Volume 28, number 3, Sept 2007; 271-277.

6. Sadler DW. The value of a thorough protocol in the investigation of sudden infant deaths. J Clin Pathol 1998;51;689-694. 
7. Iyasu S, Rowley D, Hanzlik R. Guidelines for death scene investigation of sudden, unexplained infant deaths: recommendations of the inter-agency panel on sudden infant death syndrome. MMWR Morb Mortal Wkly Rep. 1996;45:1-6.

8. Pasquale-Styles MA, Tackitt PL, Schmidt CJ. Infant death scene investigation and the assessment of potential risk factors for asphyxia: A review of 209 suddden unexpected infant deaths. J Forensic Sci, July 2007. Vol 52, no 4; 924-929.

9. Rambaud C, Guilbert M, Briand E, et al. Microbiology in sudden infant death syndrome (SIDS) and other childhood deaths. FEMS Immunology and Medical Microbiology 25 (1999); 59-66.

10. Fernandez-Rodriquez A, Ballesteros S, De Ory F et al. Virological analysis in the diagnosis of suddem children death: A medico-lagal approach. Forensic Science International 161 (2006); 8-14.

11. Bajanowski T, Vege A, Byard RW, et al. Sudden infant death syndrome (SIDS) Standardised investigations and classifications: Recommendations. Forensic Science Internataional 165 (2007); 129-143.

12. Pakis I, Karapirli M, Karayel F, et al. Quality assessment of perinatal and infant postmortem examinations in Turkey. J Forensic Sci, September 2008, Vol 53 no 5; 11661168.

13. Sharma BR. Sudden infant death syndrome - a subject of medicolegal research. The American Journal of Forensic Medicine and Pathology, Vol 28 number 1, March 2007; 69-72.

14. Randall BB, Wadee SA, Sens MA, Kinney HC, Folkerth RD, Odendaal HJ, Dempers JJ. A practical classification schema incorporating consideration of possible asphyxia in cases of sudden unexpected infant death. Forensic Sci Med Pathol. 2009; May 31.

15. Krous HF, Nadeau JM, Silva PD, Blackbourne BD. A comparison of respiratory symptoms and inflammation in sudden infant death syndrome and in accidental or inflicted infant death. Am J Forensic Med Pathol. 2003 Mar;24(1):1-8.

16. www.statssa.gov.za (Publications P03022009).

17. Mortality and causes of death in South Africa, 2006: Findings from death notification. P0309.0. Publication from Statssa. 